Research Article

\title{
An Observational Study of JE Cases Detected in National Centre for Disease Control from January 2017 to December 2019
}

\author{
Monil Singhai', Akshay C Dhariwal ${ }^{2}$, Naveen Gupta ${ }^{3}$, Sagar Goswami ${ }^{4}$, Sujeet K Singh $^{5}$ \\ ${ }^{1}$ Deputy Director, Centre for Arboviral and Zoonotic Diseases, National Centre for Disease Control, Delhi, India. \\ ${ }^{2}$ Advisor, National Vector Borne Disease Control Programme, Delhi, India. \\ ${ }^{3}$ Joint Director and Officer In-Charge, Centre for Arboviral and Zoonotic Diseases, National Centre for Disease Control, Delhi, India. \\ ${ }^{4} \mathrm{M}$.Sc. Student, Centre for Arboviral and Zoonotic Diseases, National Centre for Disease Control, Delhi, India. \\ ${ }^{5}$ Director, National Centre for Disease Control, Delhi, India. \\ DOI: https://doi.org/10.24321/0019.5138.202035
}

I $\quad \mathbf{N} \quad \mathbf{F} \quad \mathbf{O}$

\section{Corresponding Author:}

Monil Singhai, Centre for Arboviral and Zoonotic Diseases, National Centre for Disease Control, Delhi, India.

E-mail Id:

drmonil@gmail.com

Orcid Id:

https://orcid.org/0000-0003-1595-3798

How to cite this article:

Singhai M, Dhariwal AC, Gupta N, Goswami S, Singh SK. An Observational Study of JE Cases Detected in National Centre for Disease Control from January 2017 to December 2019. J Commun Dis 2020; 52(4): 12-16.

Date of Submission: 2020-11-25

Date of Acceptance: 2020-12-15

\section{$\begin{array}{llllllll}\mathbf{A} & \mathbf{B} & \mathbf{S} & \mathbf{T} & \mathbf{R} & \mathbf{A} & \mathbf{C} & \mathbf{T}\end{array}$}

Introduction: Japanese Encephalitis (JE), a leading cause of AES is a vector-borne disease is caused by the JE virus belonging to the genus Flavivirus (Flaviviridae family), and transmitted by bite of Culex vishnui subgroup tritaeniorhynchus, the most important reported vector species for JE transmission in India.

Methodology: A study was conducted on 1131 AES suspected cases in JE laboratory of Centre for Arboviral and Zoonotic diseases, National Centre for Disease Control (NCDC), Delhi from tertiary care referral hospitals of Delhi for laboratory confirmation of JE during the period from January 2017- December 2019. CSF and/ or Serum Samples from 1131 hospitalized AES suspected cases mostly from Delhi, UP, Haryana addresses. They were tested and interpreted for IgM antibodies by the JE IgM Capture ELISA kit (National Institute of Virology, Pune). The demographic profile (age/ sex/ seasonality/ place) of JE confirmed cases were analyzed.

Result: 77 (6.8\%) out of 1131 AES suspected cases were JE confirmed. 48 patients (62.2\%) were of pediatric age group 19 (24.7\%) were of middle age adult and 10 (13.0\%) were older age adults out of $77 \mathrm{JE}$ confirmed cases.. The JE cases occurred throughout the year, though maximum cases were observed in the monsoon seasons (June-October) in our study. In our study most of the JE confirmed patients were residents of state of Delhi, Uttar Pradesh and Haryana.

Conclusion: The study attempts to highlight the need of strengthening quality of laboratory detection of JE in AES cases, need for intensive vector control policy and re-evaluation of the policy of JE vaccination in India.

Keywords: Japanese encephalitis, Acute encephalitis Syndrome, JE vaccination, Vector Control, Surveillance 


\section{Introduction}

Acute Encephalitis Syndrome (AES) is a major health problem in India, because of the multiple etiologies involved, inadequate hospitalization/l aboratory facilities to diagnose known pathogens at peripheral level, unknown etiology in a substantial number of AES cases and availability of limited resources. ${ }^{1}$ Japanese Encephalitis (JE), a leading cause of AES is a vector-borne disease caused by the JE virus belonging to the genus Flavivirus (Flaviviridae family), which is closely related to the West Nile encephalitis virus and transmitted by bite of Culex vishnui subgroup tritaeniorhynchus, the most important vector species for JE transmission. ${ }^{2} \mathrm{JE}$ virus circulates in an enzootic cycle in pigs and Ardeidae/wading birds which serve as amplifying hosts. Due to the animal reservoirs, JEV cannot be eliminated but controlled by universal human vaccination in endemic areas. The main components of strategy for prevention and control of JE in the country are vaccination, case management, vector surveillance/control, laboratory diagnosis through sentinel sites. ${ }^{3}$ National Vector Borne Disease Control Programme (NVBDCP) has drawn up surveillance standards for the detection of JEV, recommended case definitions of JE, and set up criteria that are to be fulfilled to diagnose a case as JE. ${ }^{4}$ Laboratory confirmation is essential for the accurate diagnosis of JE and to study the demographic profile, changing seasonal trends and expansion of JE in newer areas.

\section{Materials and Methods}

A study was conducted on 1131 AES suspected cases in JE laboratory (Apex Reference Laboratory of NVBDCP) of Centre for Arboviral and Zoonotic diseases, National Centre for Disease Control (NCDC), Delhi, India from tertiary care referral hospitals of Delhi for laboratory confirmation of JE during the period from January 2017-December 2019.

CSF Samples from 1131 hospitalized AES suspected cases of various referral hospitals of Delhi-National Capital territory region (Delhi-NCR) were tested and interpreted for IgM antibodies by the JE IgM Capture ELISA kit (National Institute of Virology, Pune) as per manufacturer instructions.

The suspected case definition of AES as per NVBDCP/ IDSP guidelines ${ }^{4,5}$ was used for inclusion of AES suspected cases in our study which was as follows:

A person of any age, at any time of year with:

- $\quad$ Acute onset of fever and a change in mental status (including symptoms such as confusion, disorientation, coma, or inability to talk).

\section{AND/OR}

- New onset of seizures (excluding simple febrile seizures).
A suspected case with presence of IgM antibody to JE Virus in CSF was considered to be JE confirmed case.

The demographic profile (age/ sex/ seasonality/ place) of JE confirmed cases were analyzed and the optimum specimen (CSF or CSF and serum) for laboratory confirmation of JE was evaluated.

\section{Result}

A total of 1131 AES suspected cases from tertiary care referral hospitals of Delhi NCR were tested for JEV-specific IgM antibody in CSF. 77 (6.8\%) out of 1131 cases had shown presence of JEV-specific IgM antibody in CSF and were JE confirmed ( $95 \%$ confidence interval $5.4 \%$ to $8.4 \%$ ). Demographic details given in Table 1.

Table I.Age and Sex distribution of JE confirmed cases $(n=77)$ from January 2017 to December 2019

\begin{tabular}{|c|c|c|}
\hline $\begin{array}{c}\text { Age Group/ } \\
\text { Gender }\end{array}$ & $\begin{array}{c}\text { AES Suspected } \\
\text { (N=1131) }\end{array}$ & $\begin{array}{c}\text { JE confirmed case } \\
\mathbf{n = 7 7}(6.8 \%)\end{array}$ \\
\hline 0-15 years & 676 & $48(7.1 \%)$ \\
\hline $16-45$ years & 344 & $19(5.5 \%)$ \\
\hline $46-75$ years & 111 & $10(9 \%)$ \\
\hline Male & 691 & $47(6.8 \%)$ \\
\hline Female & 440 & $30(6.8 \%)$ \\
\hline
\end{tabular}

48 patients (62.2\%) were of pediatric age group 19 (24.7\%) were of middle age adult and 10 (13.0\%) were older age adults out of $77 \mathrm{JE}$ confirmed cases. The male to female ratio of JE confirmed cases was 1.6 (Table 1 ).

Table 2.Details of JE confirmed cases $(n=77)$

hospitalized in various hospitals (January 2017 December 2019)

\begin{tabular}{|c|c|c|}
\hline Referral Hospital & $\begin{array}{c}\text { Number of } \\
\text { hospitalized } \\
\text { patients }\end{array}$ & State \\
\hline $\begin{array}{c}\text { Atal Bihari Vajpayee } \\
\text { Institute of Medical } \\
\text { Sciences \& Dr. Ram } \\
\text { Manohar Lohia Hospital, } \\
\text { New Delhi }\end{array}$ & 19 & $\begin{array}{c}\text { Delhi, } \\
\text { Uttar } \\
\text { Pradesh } \\
\text { (UP), } \\
\text { Haryana }\end{array}$ \\
$\begin{array}{c}\text { Maulana Azad Medical } \\
\text { College \& Associated } \\
\text { Hospitals, Delhi. (Lok Nayak } \\
\text { Hospital, GB Pant Institute } \\
\text { of Postgraduate Medical } \\
\text { Education and Research) }\end{array}$ & 19 & $\begin{array}{c}\text { Delhi, } \\
\text { UP }\end{array}$ \\
\hline $\begin{array}{c}\text { Vardhman Mahavir Medical } \\
\text { College and Safdarjung } \\
\text { Hospital New Delhi }\end{array}$ & 13 & $\begin{array}{c}\text { Delhi, } \\
\text { UP, } \\
\text { Haryana }\end{array}$ \\
\hline \multicolumn{2}{|c}{} & \\
\hline
\end{tabular}




\begin{tabular}{|c|c|c|}
\hline $\begin{array}{l}\text { Lady Hardinge Medical } \\
\text { College \& Associated } \\
\text { Hospitals, Delhi. (Kalawati } \\
\text { Saran Children's Hospital \& } \\
\text { Sucheta Kriplani Hospital) }\end{array}$ & 10 & $\begin{array}{l}\text { Delhi, } \\
\text { UP }\end{array}$ \\
\hline $\begin{array}{l}\text { University College of } \\
\text { Medical Sciences \& Guru } \\
\text { Teg Bahadur Hospital, Delhi }\end{array}$ & 7 & $\begin{array}{l}\text { Delhi, } \\
\text { UP }\end{array}$ \\
\hline $\begin{array}{l}\text { Pandit Bhagwat Dayal } \\
\text { Sharma Post Graduate } \\
\text { Institute of Medical } \\
\text { Sciences, Rohtak, Haryana }\end{array}$ & 2 & Haryana \\
\hline St Stephen's Hospital, Delhi & 2 & $\begin{array}{l}\text { Delhi, } \\
\text { UP }\end{array}$ \\
\hline $\begin{array}{l}\text { All India Institue of Medical } \\
\text { Sciences Delhi }\end{array}$ & 2 & $\begin{array}{l}\text { Delhi, } \\
\text { UP }\end{array}$ \\
\hline $\begin{array}{l}\text { Deen Dayal Upadhyay } \\
\text { Hospital, Delhi }\end{array}$ & 2 & Delhi \\
\hline Hindu Rao Hospital, Delhi & 1 & Delhi \\
\hline
\end{tabular}

Blood samples of 21 out of $77 \mathrm{JE}$ confirmed cases were also tested for presence of JEV-specific IgM antibody. 16 patients out of 21 (76\%) JE confirmed cases had shown presence of JEV-specific IgM antibody in their blood samples, however it was not detected in blood sample of 5 patients (24\%).

The details of $77 \mathrm{JE}$ confirmed cases hospitalized in various referral hospitals of Delhi and Haryana are shown in Table 2.

JE cases were detected all-round the year, although maximum cases were observed in the monsoon seasons as shown in Figure 1.

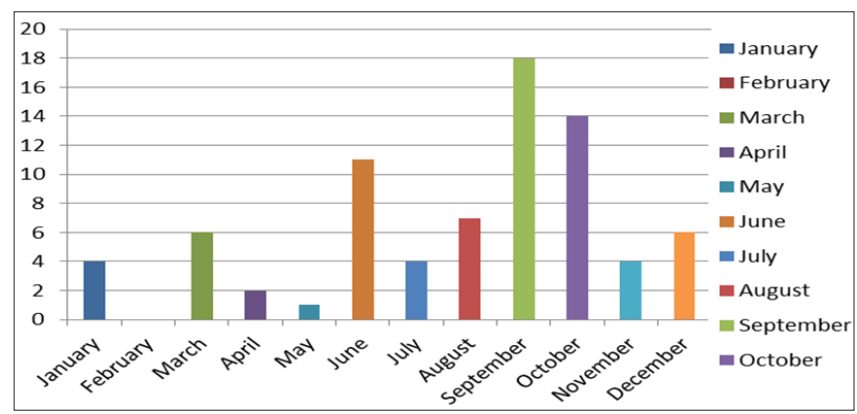

Figure I. Seasonal trends of JE confirmed cases $(n=77)$ from January 2017 to December 2019

\section{Discussion}

$\mathrm{JE}$ virus is a mosquito-borne flavivirus, and dengue, yellow fever and West Nile viruses also belong to same genus. ${ }^{6}$ The incubation period of JE is 4-14 days. Symptoms range from non-specific febrile illness to severe meningoencephalitis, occurring in about one out of 300 cases. In our study 6.8
$\%$ of AES suspected cases were JE confirmed cases. There are many possible causes of acute encephalitic syndrome; thus, laboratory confirmation is essential for the accurate diagnosis of JE. The laboratory confirmation of JE along with clinical sign/ symptoms of AES is crucial due to the cross-reactivity of antibodies to other flaviviruses in some cases. As most Acute Encephalitis Syndrome (AES) cases will require hospitalization, hence it is necessary that case-based surveillance for JE with laboratory confirmation should be the priority, such as the capacity to diagnose JE of admitted AES cases. ${ }^{3} \mathrm{CSF}$ is the preferred specimen for JE diagnosis, as IgM in serum elicited against other flavivirus infections does not cross into the CSF. Also, in cases of neuroinvasive disease, antibodies in serum may not be the cause of the neurological disease. ${ }^{7}$ In our study 5 out of 21 (34\%) JE confirmed cases did not show presence of JEV-specific IgM antibody in their blood samples. Hence, testing of CSF sample is must with or without the serum to reduce falsepositivity rates from previous infection, vaccination or to rule out cross-reaction with other flaviviruses. ${ }^{8}$ Virus culture/ Plaque reduction neutralization test is recommended for ruling out cross infections.

In our study the occurrence of JE in patient aged 0-15 years $(62.2 \%)$ was found to be higher followed by $16-45$ middle age adult (24.7\%) and in older age adults (13.0\%). JE primarily affects children $<15$ year of age but can practically occur at all ages, particularly when the virus is introduced in new areas or susceptible population where the population has no pre-existing immunity. ${ }^{8}$ In endemic areas, nearly all residents have sustained infection by young adulthood. Most adults in endemic countries have natural immunity after childhood infection, but individuals of any age may be affected. There is increasing evidence of occurrence of JE in older age group in recent times. ${ }^{9}$ This has occurred in many places where mass vaccination was carried out in children below 15 years of age. ${ }^{10}$ Similar age shifts have been observed earlier in areas with good quality, longstanding childhood JE vaccination programmes and from other Asian countries including Bangladesh, Indonesia, Thailand and Singapore and needs to be understood in detail. ${ }^{11}$ There is preliminary observation that JE should be kept in differential diagnosis of both children \& adult AES.

$\mathrm{JE}$ transmission intensifies during the rainy season, during which vector populations' increase. In the subtropics and tropics, seasonal transmission varies with monsoon rains and irrigation practices and may be prolonged or even occur year-round. The postulated explanations for JE virus transmission all-round the year and its spread in newer areas may be due to change in bird migration practices, introduction of irrigation projects for paddy farming, animal smuggling, vector adaptation and global warming etc. ${ }^{12,13}$ The ecological niche like large perennial lakes, swamps, and rice fields provide a staging ground for several migratory 
waterfowl; such areas also favor breeding and survival of mosquitoes and are conducive for JE transmission. Information Education and Communication activities play crucial role in limiting transmission.

The cases of AES and JE occurred throughout the year, though maximum cases were observed in the monsoon seasons (June-October) in our study. In our study the patients who were JE confirmed were mostly residents Delhi-NCR who had travel history of endemic states or were migrants from states such as UP, Haryana and Bihar. The occurrence of JE cases in Haryana maybe correlated with newer agricultural development and intensive rice cultivation practices supported by irrigation programs in recent years or vector adaptation including emergence of secondary/ regional vectors. Surveillance of vector Culex species and effectiveness of disinfectants will add to control of JEV in mosquito population.

Recognizing the importance of high coverage of childhood immunization interalia gravity of JE vaccination in the country Ministry of Health and Family Welfare (MoHFW) Government of India launched Mission Indradhanush in December, 2014 which focused on interventions to improve full immunization coverage for children in India from 65\% in 2014 to more than $90 \%$ by 2020 for JE in endemic districts. ${ }^{14}$ It was also recommended to introduce JE vaccination in adult populations in endemic districts based on available evidence of substantial adult disease burden.

JEV has five genotypes and each genotype exhibits unique geographical distribution characteristics, but all are capable of causing disease epidemics. ${ }^{15}$ The geographic distribution of these genotypes is not fixed. For example, JEV G1 is becoming more and more widespread and $\mathrm{G} 3$ is no longer confined to the known endemic areas of JEV. JE vaccines currently used are based on JEV strains isolated $>50$ years ago, representing only a single genotype (GIII), which no longer constitutes the dominant JEV type in many areas, hence protective efficacy of the current vaccines against the various circulating genotypes in view of changing epidemiology also needs to be studied ${ }^{15-17}$ and reconfirmed for JE vaccination to remain as mainstay in control of AES cases.

The changing demographic profile, seasonal trends and spread in newer areas of JE cases warrants an urgent need of strengthening quality surveillance along with availability of diagnostic facility at surveillance sites for estimation of JE prevalence, intensive vector control policy and reevaluation of the policy of JE vaccination in the country in view of newer evidences.

\section{Conflict of Interest: None}

\section{References}

1. Narain JP, Dhariwal AC, Maclntyre CR. Acute encephalitis in India: An unfolding tragedy. Indian J Med Res 2017; 145(5): 584-7.

2. Van den Hurk AF, Ritchie SA, Mackenzie JS. Ecology and geographical expansion of Japanese encephalitis virus. Annu Rev Entomol 2009; 54: 17-35.

3. Japanese encephalitis, Vaccine preventable disease, Surveillance Standards. 2018. Available from: https://www.who.int/immunization/ monitoring_surveillance/burden/vpd/WHO_ SurveillanceVaccinePreventable_10_Japanese Encephalitis_BW_R1.pdf?ua=1.

4. Operational Guidelines: National Programme for Prevention and Control of Japanese Encephalitis/ Acute Encephalitis Syndrome. Government of India Ministry of Health \& Family Welfare Directorate General of Health Services National Vector Borne Disease Control Programme, Delhi, India. 2014. Available from: https://nvbdcp.gov.in/WriteReadData/I892s/JE-AESPrevention-Control (NPPCJA).pdf.

5. Case definition -P form. 2019. Available from: https:// idsp.nic.in/showfile.php?lid=4262.

6. Huang YJ, Higgs S, Horne KM et al. Flavivirus-mosquito interactions. Viruses 2014; 6(11): 4703-4730.

7. Solomon T, Dung NM, Kneen $\mathrm{R}$ et al. Japanese encephalitis. Journal of Neurology, Neurosurgery \& Psychiatry 2000; 68: 405-15.

8. Johnson BW, Goodman $\mathrm{CH}$, Jee $\mathrm{Y}$ et al. Differential diagnosis of Japanese Encephalitis Virus infections with the Inbios JE Detect ${ }^{\mathrm{TM}}$ and DEN Detect ${ }^{\mathrm{TM}}$ MAC-ELISA Kits. Am J Trop Med Hyg 2016; 94(4): 820-8.

9. Tiwari S, Singh RK, Tiwari R et al. Japanese encephalitis: a review of the Indian perspective. Braz J Infect Dis 2012; 16(6): 564-573.

10. Borah J, Dutta P, Khan SA et al. A comparison of clinical features of Japanese encephalitis virus infection in the adult and pediatric age group with Acute Encephalitis Syndrome. J Clin Virol 2011; 52(1): 45-49.

11. Arai S, Matsunaga Y, Takasaki T et al. Vaccine preventable diseases surveillance program of Japan, Japanese encephalitis: surveillance and elimination effort in Japan from 1982 to 2004. Jpn J Infect Dis 2008; 61: 333-8.

12. Muniaraj M, Rajamannar V. Impact of SA 14-14-2 vaccination on the occurrence of Japanese encephalitis in India. Hum Vaccin Immunother. 2019; 15(4): 834-40.

13. Kabilan L, Rajendran R, Arunachalam N, et al. Japanese encephalitis in India: an overview. Indian J Pediatr 2004; 71(7): 609-15.

14. Kulkarni R, Sapkal GN, Kaushal H et al. Japanese Encephalitis: a brief review on Indian perspectives. Open Virol J 2018; 12: 121-30.

15. Gao X, Liu H, Li X et al. Changing Geographic Distribution of Japanese Encephalitis Virus Genotypes, 1935-2017. 
Vector-Borne and Zoonotic Diseases 2019; 19(1): 35-44.

16. Erra EO, Askling HH, Yoksan S et al. Cross-protective capacity of Japanese encephalitis (JE) vaccines against circulating heterologous JE virus genotypes. Clin Infect Dis 2013; 56(2): 267-70.

17. Connor B, Bunn WB. The changing epidemiology of Japanese encephalitis and New data: the implications for New recommendations for Japanese encephalitis vaccine. Trop Dis Travel Med Vaccines 2017; 3: 14. 